Estudios Públicos 163 (202I), I59-165

DOI: https://doi.org//0.38178/07/83089//0082/0723

Simposio

\title{
La rana, la mosca y el ser humano
}

\author{
Dirk Baecker \\ Universität Witten/Herdecke, Alemania
}

$\mathrm{H}$ umberto Maturana Romesín aceptó la invitación a la Facultad de Sociología de la Universidad de Bielefeld en el semestre de invierno 1986/87, pero estaba molesto. ¿Cómo podía Niklas Luhmann, quien lo había invitado, apropiarse indebidamente de su concepto de autopoiesis, la autoproducción de la vida a partir de la vida, para la descripción de los sistemas sociales? El mensaje de este concepto era claro. Publicado en 1970 con el título de 'Biología de la cognición' (Maturana 1970), la idea apareció el mismo año en que Salvador Allende fue electo democráticamente presidente en Chile. Maturana había estudiado medicina ahí desde 1948, antes de partir a la Universidad de Harvard para realizar sus estudios doctorales en 1956, con el fin de investigar cuestiones de anatomía y visión de las ranas. Entre otras cosas, Maturana descubrió que, tras ciertas intervenciones en su sistema nervioso, la rana lanzaba obstinadamente su lengua en una dirección cuando intentaba atrapar una mosca, mientras que la mosca se encontraba en otra distinta. No es

DiRk BAECKER (Prof. Dr.) es sociólogo y profesor de teoría cultural y management en la Universidad de Witten/Herdecke, Alemania. Estudió teoría de sistemas con Niklas Luhmann y recibió su doctorado y habilitación en la Universidad de Bielefeld, Alemania. Cuenta con numerosas publicaciones en teoría sociológica, teoría de la cultura, sociología económica y estudios organizacionales. Sus últimas publicaciones son 4.0 or Die Lücke die der Rechner lässt (Merve, 2018), Intelligenz, künstlich und komplex (Merve, 2019), Wozu Wirtschaft? (Metropolis, 2020). En español apareció recientemente su libro Estudios acerca de la próxima sociedad (Santiago: Metales Pesados, 2019). Dirección: Fakultät für Wirtschaft und Gesellschaft, Department für Philosophie, Politik und Ökonomik, Alfred-Herrhausen-Straße 50, 58448 Witten, Deutschland. Email: Dirk.Baecker@uni-wh.de.

Algunos pasajes de este artículo fueron publicados en Frankfurter Allgemeine Zeitung en la edición del 7 de mayo de 2021. 
la mosca, concluyó, sino el cerebro el que coordina las percepciones y los movimientos de la rana. En 1960 se incorporó a la Facultad de Medicina de la Universidad de Santiago de Chile, y amplió sus investigaciones hacia una teoría del organismo, la percepción y la cognición. Ocho años más tarde, Heinz von Foerster lo invitó a la Universidad de Illinois, donde fue profesor visitante hasta 1970.

Maturana entendía su biología de la cognición —una teoría del conocimiento como proceso biológico - a la vez como una filosofía de la libertad. Nadie puede privar al organismo de la autonomía de su percepción del mundo, por mucho que esta autonomía dependa de un entorno adecuado. Heinz von Foerster reconoció la gran importancia de este descubrimiento. Se adecuaba a su intento de formular una epistemología que situaba al observador en el centro de interés. "Todo lo que se dice, lo dice un observador", había escrito Maturana en el libro fundamental de la ciencia. "Todo lo que se dice, se dice a un observador", añadió von Foerster. Al mismo tiempo, Allende invitó a Stafford Beer a Chile para transformar la recién desarrollada cibernética del observador en un sistema de monitoreo social de la economía chilena. ${ }^{1}$ Poco tiempo después de los turbulentos años de la década de 1960, las esperanzas de que por fin se estableciera una sociedad libre y autodeterminada debían ser abrumadoras. Sabemos cómo terminó aquello. Apoyados por la CIA, primero los empresarios del cobre, luego los transportistas y los camioneros entraron en huelga paralizando el país. En 1973, Pinochet dio un golpe de Estado y estableció una dictadura militar.

Maturana debió seguir todo esto con la mayor preocupación. Continuó con sus clases. En algún momento dedicó un semestre entero a la pregunta por una teoría del organismo. Al final de ese semestre, un estudiante se le acercó y le habría dicho que ahora sabía todo sobre la producción de proteínas a partir de proteínas, pero que todavía no entendía qué era la 'vida'. Maturana no sabía la respuesta. Se dedicó a responder esta pregunta durante las vacaciones y encontró la respuesta en su concepto de autopoiesis. La autopoiesis, la autoproducción, es un proceso en el que, desde los componentes de un ser vivo, principalmente células y proteínas, se obtienen no solo los componentes del ser vivo, sino también la red de producción de los componentes del ser vivo. La

\footnotetext{
${ }^{1}$ Al respecto se puede consultar E. Medina, Revolucionarios cibernéticos. Tecnología y política en el Chile de Salvador Allende (Santiago: LOM Ediciones, 2013). [N. del T.]
} 
vida es el despliegue de una tautología. Es una invención única que se ha repetido en infinidad de formas desde que existe la vida en la Tierra. La vida, según esta consideración, no puede explicarse por su origen, sino solo como continuidad. Es posible especular sobre los procesos que precedieron a la aparición de la vida a partir de una sopa primordial, pero el punto crucial no es su surgimiento, sino su reproducción. La vida es su propia (autos) obra (poiesis).

A los turbulentos años sesenta les siguieron los grises setenta. Maturana continuó desarrollando su teoría junto con Francisco J. Varela y se hizo famoso por su intento de confirmar empíricamente el concepto de autopoiesis para los casos del sistema nervioso conformado por impulsos y del organismo conformado por células. Clausura operativa era la palabra clave del momento. Finalmente se comprendía lo que la neurofisiología de Johannes Müller, Gustav Theodor Fechner y Hermann von Helmholtz ya había supuesto en el siglo XIX, sin poder enfrentar realmente esta idea. El sistema nervioso no es nada más que sus impulsos. Debe construir el conjunto de figuras, colores, sonidos, sensaciones y representaciones de las que trata la conciencia a partir de esos impulsos y nada más que de esos impulsos. El organismo está abierto al mundo porque trabaja cerrado sobre sí mismo. Inconcebible. La neurociencia actual aún no está a la altura de este pensamiento.

Cuando Luhmann comenzó a probar la idea de la autopoiesis en los sistemas sociales en la década de 1980, Maturana sintió que se lo malinterpretaba. Temía que Luhmann estuviera sometiendo de nuevo al ser humano como individuo a las mismas fuerzas sociales contra las que Maturana y Varela habían desarrollado su concepto. Que Luhmann se tomaba en serio la idea de libertad y la utilizara también para describir familias, organizaciones y sistemas funcionales, para no hablar de la propia sociedad, era imposible de transmitir a Maturana. El intento de discutir con él en Bielefeld fue rápidamente abandonado. Luhmann y Maturana debían dar en conjunto el seminario aquel invierno de 1986. Pero rápidamente Luhmann se dio cuenta de que el fuerte de Maturana no estaba en la discusión. Ya desde la segunda sesión, colocó su silla a un costado de la sala, dejó el podio a Maturana y siguió con fascinación la conferencia de un hombre que fue capaz de dedicar toda una vida académica a uno de los pensamientos más improbables que se pueden concebir. 
$\mathrm{Ni}$ antes ni después se ha podido convencer a los creadores del concepto de autopoiesis de su fecundidad en la sociología. En el volumen editado por Fritz B. Simon (1988), Lebende Systeme: Wirklichkeitskonstruktionen in der systemischen Therapie, ${ }^{2}$ aparece una conversación entre Heinz von Foerster, Niklas Luhmann y Francisco Varela en la que se pueden encontrar algunos argumentos importantes al respecto (von Foerster, Luhmann y Varela 1988).

Hasta la fecha quedan varias cuestiones por resolver en el trabajo con el concepto de autopoiesis. Una de estas se refiere a la propuesta de Luhmann de temporalizar el concepto de componente de sistemas autopoiéticos. Los componentes son eventos que diferencian y reproducen autorreferencialmente los sistemas autopoiéticos. Los eventos aparecen y desaparecen nuevamente. Tienen una existencia selectivamente estable, pero fugaz. De esta forma, reproducen el problema que resuelven. Son un evento de conexión en busca de eventos de conexión. En sociología, esto es evidente de manera inmediata. Tanto la acción como la comunicación solo pueden pensarse en este sentido temporalizado. Cualquier estructura que vaya más allá de esto es una expectativa de que ciertos eventos ocurran o no. Esta idea es compatible con el concepto de clausura operativa de von Foerster: los sistemas operacionalmente clausurados están sujetos a la condición — su única condición- de que todo final es también un principio. Esto implica una comprensión temporal del funcionamiento, aunque von Foerster no lo haga explícito. Posiblemente esto sea más fácil de entender en la física que en la biología. Los impulsos eléctricos en sí mismos tienen una identidad efímera. ¿Cómo podría comprobarse esta idea en la descripción de sistemas neuronales, sistemas inmunitarios u organismos?

Una segunda cuestión se refiere a la recepción incompleta de la idea básica de autopoiesis en la teoría de los sistemas sociales. La famosa definición formulada por Maturana (1981) en su artículo en la antología de Milan Zeleny (1981) se centra en una red de producción de componentes que 1) generan y realizan recursivamente esta red a través de su interacción, y 2) que, en el espacio en el que existen, constituyen los límites de la red que participan en la realización de esta red. La recepción de este concepto por parte de Luhmann se centra en la recursividad de los

\footnotetext{
${ }^{2}$ La traducción del título del libro es 'Sistemas vivientes: construcciones de realidad en la terapia sistémica'. [N. del T.]
} 
componentes, interpretada como recursividad y reflexividad de los eventos operativos que diferencian y reproducen un sistema autopoiético. Luhmann no abordó el concepto de red. En la década de 1980, cuando Luhmann formulaba su teoría de sistemas sociales, este no llamaba la atención. El concepto de red apenas ha tenido importancia en la sociología. En el mejor de los casos describía ciertas dinámicas de grupo en las que la teoría de sistemas tenía poco interés. Sin embargo, desde la perspectiva de la década de 1990 y del presente hay que lamentar esta omisión. Con la teoría de redes de Bruno Latour (2008) y sobre todo con la de Harrison C. White (2008), se ha desarrollado en sociología una gama de teorías que puede competir con la teoría de sistemas. Las redes son estructuras que tienen exactamente el mismo valor de describir las expectativas inciertas que Luhmann formuló para su concepto de estructura. Se podría haber mejorado considerablemente tanto el alcance empírico como la agudeza analítica de la teoría de sistemas si se hubiera incorporado, en una fase temprana, un concepto de red que, en el sentido de Maturana, permitiera describir las estructuras tan concretas como intercambiables en las que tiene lugar la autopoiesis de las operaciones basales de los sistemas sociales, la que empíricamente sigue siendo algo abstracta.

Una tercera cuestión refiere al concepto de producción. Luhmann ha señalado que la autopoiesis debe entenderse como producción precisamente en el sentido de que, fuera de los contextos técnicos, toda producción siempre tiene que ver con la combinación de factores de producción disponibles y no disponibles. En sociología, esto remite al conocimiento y al no-conocimiento, ambos subyacentes a toda comunicación, ya que de otro modo no habría razón para la comunicación. Pero, ¿qué significa aquí 'producción'? La autopoiesis de los sistemas sociales se hace posible, como dice Luhmann, desde atrás. Es una producción fundamentalmente retrospectiva [nachträglich]. Urs Stäheli (2000) ha señalado el importante papel que desempeña el concepto de retrospectividad en el psicoanálisis de Freud. La retrospectividad pasa por alto, pero rescata y reinterpreta cada momento actual de producción de una acción o comunicación. ¿Qué significa esto para los sistemas sociales? ¿Producen estos esa mínima complicación de una descripción que el sistema no logra expresar uno-a-uno y que John von Neumann (1966) ya describió como condición elemental de una reproducción inteligente en tanto creativa? 
Y, desde luego, el último tópico que podría haberse tratado si hubiese existido un mejor intercambio entre las teorías biológicas y sociológicas de la autopoiesis se refiere a la cuestión de si las matemáticas son adecuadas para modelar los sistemas autopoiéticos y, en caso afirmativo, qué matemáticas. Varela (1979) jugó con la idea de volver a Laws of Form de George Spencer-Brown (1969). Curiosamente, complementó el cálculo de indicaciones justamente en torno al valor autónomo que este ya posee en la reentrada de la distinción. Pero incluso esto habría permitido una conversación con Luhmann, cuya comprensión de la comunicación en sus trabajos más tardíos (Luhmann 1997) se orientó cada vez más a la idea de Spencer-Brown de operaciones de distinción con capacidad de reentrada [re-entry]. ¿Puede entenderse la idea de autopoiesis en términos de teoría de la forma — ver Baecker (2013)? ¿Es factible que el estado no marcado [unmarked state] de las operaciones de producción de proteínas también aporte un direccionamiento tal como lo hacen los impulsos neuronales o los actos comunicativos? ¿Están vinculadas la recursividad y la reflexividad de las operaciones autopoiéticas en cada tipo de sistema autorreferencial a cada estado de indeterminación [state of indeterminacy] en el que las operaciones comienzan a oscilar, a recordar y a modular su red? ${ }^{3}$

Todas estas cuestiones surgen de un programa de investigación que toma en serio la diferencia empírica entre sistemas orgánicos, mentales y sociales, así como la posibilidad de su comparación desde el punto de vista de su autopoiesis. Tal vez haya llegado el momento de llevar a cabo un programa de investigación de este tipo. En la década de 1980 prevalecía el fastidio ante la importación sociológica del concepto y, además, todos estaban demasiado ocupados en reproducir, en cada sistema social, la idea de una autopoiesis de los sistemas sociales que surge de sus propios eventos. Sin embargo, esto no debe impedir que, medio siglo después de su formulación, sigamos concibiendo el extraordinario impulso de Maturana y Varela como una obra prometedora.

Tras su emeritación, Maturana, en conjunto con Ximena Dávila, fundaron el Instituto Matríztica en Santiago de Chile, que se ocupa de los temas del amor y el juego como "fundamentos olvidados del ser humano",

\footnotetext{
${ }^{3}$ Para un acceso introductorio a estos problemas lógicos y a la teoría de sistemas sociales en general, ver N. Luhmann, Introducción a la teoría de sistemas (México DF: Universidad Iberoamericana, 2002). [N. del T.]
} 
según indica el título de un libro (Maturana y Verden-Zöller 2016), y que, entre otras cosas, busca responder a la pregunta de por qué las formas matriarcales de convivencia se han establecido tan pocas veces en la historia de la humanidad. Maturana respondió la pregunta qué es la vida. La pregunta qué es el conocimiento nunca lo abandonó. Esto equivalía a una teoría del observador sobre la que una vez planeó escribir un libro junto con von Foerster. Este libro nunca llegó a existir. Solo el título estaba aparentemente claro: 'Autopoiesis de la autopoiesis'. Maturana murió en Santiago de Chile el 6 de mayo de 2021.

(Traducción del alemán de Aldo Mascareño)

\section{Bibliografía}

Baecker, D. 2013. A Calculus for Autopoiesis (249-267). En Baecker, D. y Priddat, B. (eds.), Ökonomie der Werte: Festschrift zum 65. Geburtstag von Michael Hutter. Marburg: Metropolis. Disponible en: https://ssrn.com/abstract=2073362 [26 de julio 2021].

Latour, B. 2008. Reassembling the Social: An Introduction to Actor-Network-Theory. Oxford: Oxford University Press.

Luhmann, N. 1997. The Control of Intransparency. Systems Research and Behavioral Science 14, 359-371.

Maturana, H. 1970. Biology of Cognition. Biological Computer Laboratory Research Report BCL 9.0. Urbana, IL: University of Illinois.

Maturana, H. 1981. Autopoiesis (21-33). En Zeleny, M. (ed.), Autopoiesis: A Theory of Living Organizations. New York: Elsevier.

Maturana, H. y Verden-Zöller, G. 2016. Amor y juego: fundamentos olvidados de lo humano. México DF: JC Saez Editor.

Simon, F. (ed.) 1988. Wirklichkeitskonstruktionen in der systemischen Therapie. Berlin, Heidelberg: Springer Verlag.

Spencer-Brown, G. 1969. Laws of Form. London: Allen \& Unwin.

Stäheli, U. 2000. Sinnzusammenbrüche. Weilerswist: Velbrück Wissenschaft.

Varela, F. 1979. The Extended Calculus of Indications Interpreted as a Three-Valued Logic. Notre Dame Journal of Formal Logic 20(1), 141-146.

von Foerster, H., Luhmann, N. y Varela, F. 1988. Kreuzverhör — Fragen an Heinz von Foerster, Niklas Luhmann und Francisco Varela (95-107). En Simon, F. (ed.), Wirklichkeitskonstruktionen in der systemischen Therapie. Berlin, Heidelberg: Springer Verlag.

von Neumann, J. 1966. Theory of Self-Reproducing Automata. Urbana, IL: University of Illinois Press.

White, H. 2008. Identity and Control. Princeton: Princeton University Press.

Zeleny, M. (ed.) 1981. Autopoiesis: A Theory of Living Organizations. New York: Elsevier. EP 